\title{
Environmental asbestos and pleural mesothelioma in the north-west of Pakistan; a serious health hazard with potentially fatal outcome
}

\begin{abstract}
Background: Mesothelioma was first reported in the Khyber Pakhtunkhwa (KP) in 1990's. The diagnosis was made on the basis of histopathology but no Immunohistochemical (IHC) markers were applied. So a need was felt to conduct a more authentic study to establish a cause and effect relationship between environmental asbestos exposure and mesothelioma.

Aims: Aims of the present study is to confirm the presence of mesothelioma with a more authentic diagnosis, using histopathology and IHC-markers together and to know the overall burden of mesothelioma as a cause of exudative pleural effusions.

Material and methods: It was a prospective study and conducted at the department of Pulmonology, Medical teaching Institute, Lady Reading Hospital Peshawar, KP, Pakistan. The hospital receives patients for consultation and admission from all over the KP, the Federally administrative tribal areas (FATA) and Afghanistan. The study period was three years from January 2015 to December 2017. All the patients with exudative effusions were biopsied after a verbal and written informed consent. Three or four pieces of pleural biopsies were taken on an average, using Abram's pleural biopsy needle. Ultrasound guidance was used for biopsy-site selection in cases of smaller and/or loculated effusions. Two percent (2\%) injectable lignocain was used as local anesthetic. Intravenous dormicum and/or tramadol was/were used in excessively apprehensive cases

Results: A total of 11,056 were admitted to the department during the 3 years, 6274 $(56.75 \%)$ being male and $4782(43.25 \%)$ being female. Of these, $1667(15.07 \%)$ patients had exudative pleural effusions and were biopsied. A total of $136(08.15 \%)$ cases out of all the cases undergoing pleural biopsies turned out to be malignant, out which $33(24.30 \%)$ were mesothelioma. Male to female ratio for mesothelioma was 2 ( $65.20 \%$ cases): 1 ( $34.80 \%$ cases). Age distribution showed no cases below the age 20 , $18.20 \%$ cases between the age-range $21-40$ and $81.80 \%$ cases above the age 40 . All the mesothelioma cases were histologically proven and supported by positive IHCmarkers for pleural mesothelioma and negative IHC-markers for other malignancies.

Conclusion: North-Western areas of Pakistan, the FATA and adjacent areas of the KP province, have histopathologically and immunohistochemically proven cases of pleural mesothelioma. The burden of mesothelioma is quite high, accounting for almost two percent and one-fourth of all the exudative and the malignant pleural effusions, respectively.
\end{abstract}

Keywords: asbestos, mesothelioma, malignant pleural effusion, khyber pakhtunkhwa, chrysotile, tremolite
Volume I Issue 3 - 2018

\author{
Mohammad Yousaf Khan, Zafar Iqbal, \\ Muhammad Imran \\ Department of Pulmonology, Lady Reading Hospital Peshawar, \\ Pakistan
}

Correspondence: Zafar lqbal, Department of Pulmonology, Lady Reading Hospital, Peshawar, Pakistan,

Email drzafariqbalpulmo@gmail.com

Received: April 12, 2018| Published: May 31, 2018

\section{Back ground and introduction}

Though man has been using asbestos for ages ${ }^{1,2}$ but the potentially carcinogenic airborne asbestos fibers, contaminating the environment, have been neglected till recent past. ${ }^{3,4}$ Naturally occurring asbestos deposits are located in the Himalayan zone, in the north-west of Pakistan. ${ }^{5,6}$ The major rock belts containing asbestos are in the KP province and adjacent FATA of Pakistan. Around $90 \%$ of the natural asbestos deposits of Pakistan are in the KP province and the FATA as shown in the map (Figure 1). Asbestos fibers find their way into the environment during the process of freeing it from the rocks by crushing and blowing. Asbestos also occurs, in close association, with marble in rocks. Quarrying for marble, done extensively in the KP, leads to airborne asbestos fibers in the surroundings. ${ }^{7}$ The concentration of respirable asbestos fibers in the mining fields in Pakistan are higher than the permissible limits. ${ }^{8}$ In addition to mining, there are large number of manufacturing units of local and imported asbestos in the KP. Asbestos has many commercial uses because of its long fibers and its heat and electric insulator properties. To count a few of its multiple commercial uses, asbestos is used in cement, flooring, ceiling, tiles, corrugated sheets, tapes and insulations industries. ${ }^{9}$ Moreover, asbestos is commonly used for white washing houses in the rural areas surrounding asbestos mines. Risk assessment for environmental asbestos has only been done in the FATA and the KP province of Pakistan. The first study reporting the occurrence of asbestos deposits in Pakistan was published in 1967. ${ }^{10}$ Mainly, occurrence studies have been done since then till $2004 .{ }^{11-14}$ The $1^{\text {st }}$ study 
on physical properties of asbestos deposits in Pakistan was published in 2005. Mainly 03 types of asbestos were discovered, chrysotile, tremolite and anthophyllite. Their physical dimensions were less than 3 micrometers in diameter and more than 8 micrometers in length, well within the range of asbestos fibers with carcinogenic potential. ${ }^{15}$ The dose of environmental asbestos fibers in and around the asbestos mining field was more than 100 times the permissible limit. ${ }^{9}$ Since 2009 , international standards are available for asbestos ${ }^{16,17}$ workers but workers in the KP use shovels for extraction and bare hands for spread of asbestos for drying. There is no concept of occupational safety and industrial hygiene. ${ }^{18}$ Mesothelioma was first reported in the KP in 1990's. The diagnosis was made on the basis of histopathology but no Immunohistochemical (IHC) markers were applied. ${ }^{19}$ Moreover, there was no history of asbestos exposure in the ship breaking industry. Additionally, the medical fraternity was largely unaware of the facts that mesothelioma could develop as a result of environmental asbestos exposure and that environmental asbestos exposure was very high in various parts of the FATA and the KP. Therefore the regulating fraternity needed a pinch of salt to swallow the results of the studies reporting mesothelioma secondary to asbestos exposure. With increasing awareness regarding mesothelioma developing due to environmental asbestos exposure, a need was felt to conduct a more authentic study to establish a cause and effect relationship between environmental asbestos exposure and mesothelioma.

\section{Aims}

The aim of the study was:

1. To estimate the overall burden of mesothelioma as a cause of exudative pleural effusions.

2. To confirm the diagnosis of mesothelioma using more authentic diagnostic procedures like histopathology and IHC-markers together.

\section{Inclusion criterion}

All patients with exudative pleural effusions (pleural protein $>3 \mathrm{gm} / \mathrm{dl}$, ratio of pleural protein and serum protein $>0.5$, ratio of pleural lactate dehydrogenase (LDH) level to serum LDH level $>0.6$ or pleural LDH level two thirds of the serum LDH)

\section{Exclusion criterion}

Patients lab profile with negative exudative effusion or positive transudative effusion.

\section{Material and methods}

The study was conducted at the department of Pulmonology, Medical teaching Institute, Lady Reading Hospital Peshawar and Khyber Pakhtunkhwa, Pakistan. The hospital receives patients for consultation and admission from all over the KP, the FATA and Afghanistan. The study period was three years from January 2015 to December 2017. All the patients with exudative effusions were biopsied after a verbal and written informed consent. Three or four pieces of pleural biopsies were taken on an average, using Abram's pleural biopsy needle. Ultrasound guidance was used for biopsy-site selection in cases of smaller and/or loculated effusions. Two percent $(2 \%)$ injectable lignocain was used as local anesthetic. Intravenous dormicum and/or tramadol was/were used in excessively apprehensive cases. No serious complications were observed. The commonest side effect was mild localized pain and occasional faintness in over apprehensive patients. Small pneumothorax was also observed occasionally. None of the patients needed an invasive intervention to treat any complication of the procedure. Patients with larger effusions but occupying less than half of the hemithorax were therapeutically aspirated. While patients with very large effusions, occupying more than half of the hemithorax, were also chest intubated which was used for future pleurodesis, if needed.

\section{Results}

A total of 11,056 patients were admitted to the department during the 3 years, $6274(56.75 \%)$ being male and $4782(43.25 \%)$ being female (Table 1). Of these, $1667(15.07 \%)$ patients had exudative pleural effusions and were biopsied. A total of $136(08.15 \%)$ cases out of all the cases undergoing pleural biopsies turned out to be malignant, out of which $33(24.30 \%)$ were mesothelioma (Table 2). Male to female ratio for mesothelioma was $2(65.20 \%$ cases): 1 (34.80\% cases) (Table 3). Age distribution showed no cases below the age 20, $6(18.20 \%)$ cases between the age-range 21-40, 13 (39.4\%) between 41 to 60 years of age. Fourteen $(42.4 \%)$ cases were seen above the age of 60 years (Table 4). All the mesothelioma cases were histologically proven and supported by positive IHC-markers for mesothelioma and negative IHC-markers for other malignancies. A list of various IHC markers applied to the histopathology specimens is shown in Table 5.

Table I Total no. of admissions to the department of pulmonology

\begin{tabular}{lll}
\hline Gender & N & Percent \\
\hline Male & 6274 & 56.75 \\
Female & 4782 & 43.25 \\
Total & 11056 & $100 \%$
\end{tabular}

Table 2 Overall burden of mesothelioma in patients with exudative pleural effusions

\begin{tabular}{lll}
\hline & N & Percent \\
\hline Total No. of patients with positive exudative pleural effusions (EPE) & 1667 & $15.07 \%$ of total admissions \\
Total No. of patients positive for malignancy after biopsy test & 136 & $8.15 \%$ of total cases who underwent pleural biopsy \\
Total No. of patients positive for mesothelioma among malignant cases & 33 & $24.3 \%$ of total malignant cases \\
\hline
\end{tabular}

Table 3 Gender wise distribution of Mesothelioma cases

Table 4 Age wise distribution of Mesothelioma cases

\begin{tabular}{lll}
\hline Sex & N & Percent \\
\hline Male & 22 & $65.20 \%$ \\
Female & 11 & $34.80 \%$ \\
Total & 33 & $100 \%$ \\
\hline
\end{tabular}

\begin{tabular}{lll}
\hline Age (in years) & $\mathbf{N}$ & Percent \\
\hline 1 to 20 & 0 & 0 \\
21 to 40 & 6 & 18.2 \\
41 to 60 & 13 & 39.4 \\
61 and above & 14 & 42.4 \\
Total & 33 & $100 \%$ \\
\hline
\end{tabular}

Citation: Khan MY, lqbal Z, Imran M. Environmental asbestos and pleural mesothelioma in the north-west of Pakistan; a serious health hazard with potentially fatal outcome. MOJ Curr Res \& Rev. 20I8; I(3):I24-I27. DOI: I0.15406/mojcrr.20I8.0I.000I9 
Table 5 List of IHC markers applied to the histopathology specimens

\begin{tabular}{ll}
\hline $\begin{array}{l}\text { Commonly applied } \\
\text { markers }\end{array}$ & $\begin{array}{l}\text { Uncommonly/Occasionally applied } \\
\text { markers }\end{array}$ \\
\hline Pancytokeratin & CK7 \\
Calretinin & LCA \\
WT1 & CK20 \\
TTF1 & Mesothelin \\
CK5/6 & Desmin \\
& ER \\
& CAM5.2 \\
& CD45 \\
& CD3 \\
& CD56 \\
& BerEP4 \\
\hline
\end{tabular}

\section{Discussion}

Environmental asbestos exposure has not received the attention it deserves because of its potentially fatal consequences. Asbestos exposure related to ship breaking had been the main focus of attention till a few decades ago and even recently. ${ }^{20-23}$ It was after the 1990 when mesothelioma, due to environmental asbestos exposure, was first reported form Turkey, ${ }^{24}$ other studies reported an association between environmental exposure to asbestos and mesothelioma. ${ }^{25-27}$ Many studies have reported the presence of asbestos deposits in the north-west of Pakistan. ${ }^{11-14}$ In the year 2005, a study from Mohmand Agency of the FATA, reported the presence of chrysotile, tremolite and anthophyllite asbestos deposits. This study also showed that the physical size of asbestos fibers in the area was well within the potentially carcinogenic asbestos-fiber size (i.e. within the range of less than 3.5micrometer in diameter and more than 5 micrometer in length). The dose of the airborne asbestos fibers was more than 100 -times the permissible level of $0.1 \mathrm{f} / \mathrm{cc} .{ }^{15} \mathrm{~A}$ cause and effect relation between environmental asbestos exposure and mesothelioma in the FATA and the KP province of Pakistan was first established in the 1990s and was published in the year 2001. ${ }^{19}$ The histological diagnosis of mesothelioma reported in this study was questioned because IHC-makers were not applied. Biopsy specimens obtained via closed pleural biopsies are small and pose difficulty in reaching a definitive diagnosis of mesothelioma. Moreover, the three histological types of mesothelioma; namely epithelioid, sarcomatoid and mixed variety can resemble other malignancies. This issue can be resolved by simultaneously applying positive IHC-markers for confirmation of mesothelioma and negative IHC-markers for ruling out other malignancies. ${ }^{28-33}$ In this study, various IHC-markers were applied to the histopathology specimens.

Thus a definite diagnosis of mesothelioma was made and a cause and effect relationship between environmental asbestos exposure and mesothelioma was reconfirmed. The overall burden of mesothelioma was found to be high, almost one-fourth $(24.30 \%)$ of all the malignant pleural effusions. Any other study mentioning burden of mesothelioma as percent of malignant pleural effusion could not be found for comparison, both nationally and internationally. Traditionally, the grown up females belonging to the rural population of the FATA and the KP province are house bound, most of the time. The male to female ratio of 2:1 for mesothelioma strongly suggest the presence of airborne asbestos in the houses and villages around asbestos mines. No mesothelioma under the age of 20 years, less than $20 \%$ between the ages $21-40$ years and more than $80 \%$ above the age of 40 years, highlights the long latent period between asbestos exposure and development of mesothelioma. The latent period usually ranges between 20 to 40 years and sometimes up to 60 years. ${ }^{34-36}$ The overall burden of mesothelioma as a cause of exudative pleural effusion and malignant pleural effusion might have been under-estimated in the study. Various geological and environmental surveys have reported asbestos deposits and environmental asbestos exposure in certain specific parts of the KP and the FATA ${ }^{5-15}$ as shown in the map. Rest of the KP and the FATA, include rocky, plain and sandy areas. These areas have not been surveyed and may or may not have asbestos deposits and sources of asbestos exposure. If cases of effusion from areas with no reported asbestos deposits and asbestos exposure are excluded from the study, the total number of mesothelioma as percentage of total exudative effusions and as percentage of total malignant effusions might increase well above the current reported Figure 1 of $1.98 \%$ and $24.30 \%$, respectively.

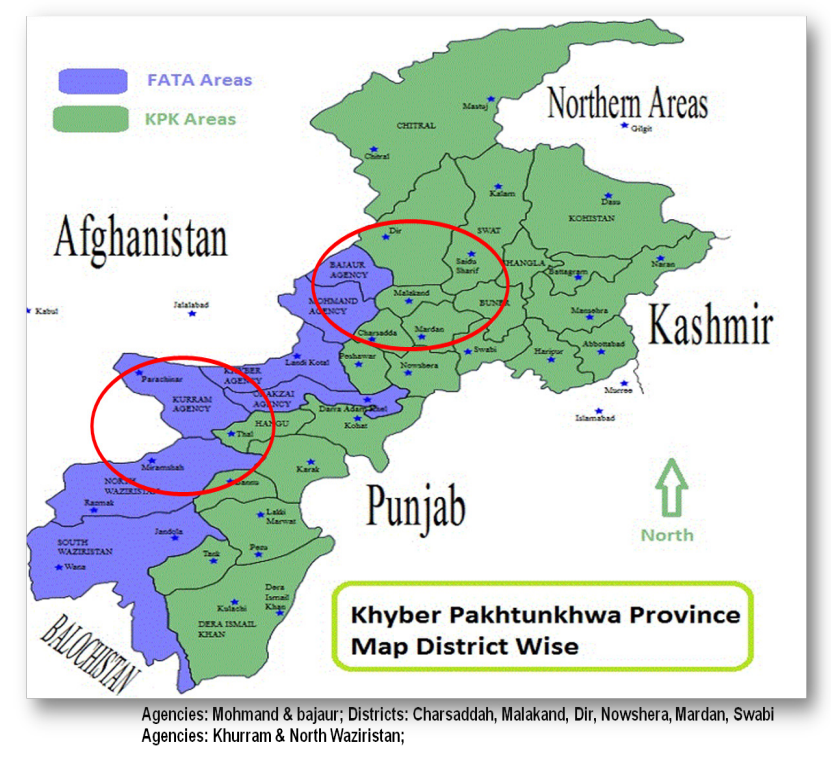

Figure I Map showing areas of KP province (formerly known as North West Frontier province) in Pakistan. Asbestos occurrence shown in red circles.(Source: physical maps of Pakistan, Ezilon map).

\section{Conclusion}

North-Western areas of Pakistan, the FATA and adjacent areas of the KP province, have histopathologically and immunohistochemically proven cases of pleural mesothelioma. The burden of mesothelioma is quite high, accounting for almost two percent and one-fourth among all the exudative and the pleural effusion patients, respectively.

\section{Acknowledgments}

We acknowledge the contributions of Mr. Mazhar Ali Khan and Muhammad Usman Ali for their computer assistance and Staff of Pulmonology department Lady Reading Hospital Peshawar, Pakistan for maintaining all the records. 


\section{Conflict of interest}

Author declares that there is no conflict of interest.

\section{References}

1. Mike Searle, Dilek Y, Newcomb S. Ophiolite Concept and the Evolution of Geological Thought. Boulder, Geological Society of America. Geological Magazine. 2004;141(3):390-391.

2. Rapp GR. Archaeomineralogy. Springer: Berlin; 2009.

3. IARC Working Group on the Evaluation of Carcinogenic Risks to Humans. Arsenic, Metals, Fibers and Dusts. Lyon. IARC Monogr Eval Carcinog Risks Hum. 2012;100(Pt C):11-465.

4. Robinson BW, Musk AW, Lake RA. Malignant mesothelioma. Lancet. 2005;366(9483):397-408.

5. Jehan N, Hamidullah S. Lung Fibrosis and asbestos in Northern Pakistan. In: Sobel Appel Strecker M, et al. editors. Terra Nostra Schriften der Alfred Wegener Stiftung. 1999. p. 76-7.

6. Jehan N, Ahmad I. Petrochemistry of asbestos bearing rocks from Skhakot-Qila Ultramafic Complex, northern Pakistan. Journal of Himalayan Earth Sciences. 2006;39:75-83.

7. Hamidullah S. Asbestos occurrences in Mohmand Agency: Genesis, economics, and related health hazards. Geological Bulletin University of Peshawar. 1984;17:207-220.

8. Iftikhar S, Ali M, Nergis Y. Risks and Hazards Study of Asbestos in Pakistan. Int J econ environ geol. 2015;6(1):25-28.

9. Jehan N. Sustainable management of mineral resources with special reference to asbestos and silica in Northern Pakistan. National Center of Excellence in Geol Univ Peshawar HEC. 2004.

10. Qaisar MA, Khan AH. Mineralogy of asbestos from Kurram Agency Pakistan. J Sci Indus Res. 1967;12:163-4.

11. Qaisar MA, Ali K, Khan AH. Mineralogy of asbestos from north-west Pakistan. J Sci Indus Res. 1967;116-120.

12. Rafiq M, Shah MT, Ahmad I. Note on tremolite zone from the extension of Shkakote-Qila Ultramafic complex in Utmankhel, Mohmand Agency. Geol Bull Univ Peshawar. 1984;17:178-9.

13. Rafiq M. Extension of Shkakot Qila ultramafic complex in Utman khel, Mohmad Agency, NWFP, Pakitan. Geol Bull Univ Peshawar. 1984;17:53-59.

14. Jehan N. Asbestos risks: occupational and paraoccupational health status in Pakistan. Global asbestos conference. 2004.

15. Jehan N, Ahmed I. Fibrogenic and carcinogenic characteristics of Asbestos occurring in Mohmand Agency. Northern Pakistan: Geol Bull Univ Peshawar. 2005.

16. Mikelis NE. A statistical overview of ship recycling. WMU J Maritime Affairs. 2008;7(1):227-39.

17. World Bank. Good practice note: Asbestos: Occupational and community health issues. 2009.

18. Jehan KH, Khan AN. Spheroidal asbestos from Qila, Charsadda Tehsil Pakistan. JSIR. 1963;16:266-7.

19. Javaid A, Ullah Z. Mesothelioma in NWFP, Pakistan. Pak J Chest Med. 2001;7(1).
20. Hayman B, Dogliani M, Kvale I, et al. Technologies for reduced environmental impact from ships-Ship building, maintenance and dismantling aspects. ENSUS. 2000.

21. Andersen AB. Worker safety in the ship-breaking industries. International Labour Office Geneva. 2001.

22. Abdi R. India's ship-scrapping industry: monument to the abuse of human labour and the environment. IIAS Newsletter. 2003;32(46).

23. Sonak S, Sonak M, Giriyan A. Shipping hazardous waste: implications for economically developing countries. International Environmental Agreements: Politics, Law and Economics. 2008;8(2):143-59.

24. Metintas M, Hillerdal G, Metintas S. Mesothelioma due to environmental exposure to erionite: follow-up of a Turkish emigrant cohort. Eur Respir J. 1999;13(3):523-6.

25. Şenyiğit $A$, Babayiğit $C$, Gökirmak $M$, et al. Incidence of malignant pleural mesothelioma due to environmental asbestos fiber exposure in the southeast of Turkey. Respiration. 2000;67(6):610-4.

26. Magnani C, Agudo A, Gonzalez CA, et al. Multicentric study on malignant pleural mesothelioma and non-occupational exposure to asbestos. Br J Cancer. 2000;83(1):104-11.

27. Luo S, Liu X, Mu S, et al. Asbestos related diseases from environmental exposure to crocidolite in Da-yao, China. I. Review of exposure and epidemiological data. Occup Environ Med. 2003;60(1):35-42.

28. Maule MM, Magnani C, Dalmasso P, et al. Modeling mesothelioma risk associated with environmental asbestos exposure. Environ Health Perspect. 2007;115(7):1066-71.

29. Husain AN, Colby TV, Ordóñez NG, et al. Guidelines for pathologic diagnosis of mesothelioma: a consensus statement from the International Mesothelioma Interest Group. Arch Pathol Lab Med. 2009;133(8):1317-31.

30. Ordonez NG. The immunohistochemical diagnosis of mesothelioma: a comparative study of epithelioid mesothelioma and lung adenocarcinoma. The Am J Surgical Pathol. 2003;27(8):1031-51.

31. Ordonez NG. What are the current best immunohistochemical markers for the diagnosis of epithelioid mesothelioma? A review and update. Hum Pathol. 2007;38(1):1-6.

32. King JE, Thatcher N, Pickering CA, et al. Sensitivity and specificity of immunohistochemical markers used in the diagnosis of epithelioid mesothelioma: a detailed systematic analysis using published data. Histopathology. 2006;48(3):223-32.

33. Lucas DR, Pass HI, Madan SK, et al. Sarcomatoid mesothelioma and its histological mimics: a comparative immunohistochemical study. Histopathology. 2003;42(3):270-9.

34. Frost $\mathrm{G}$. The latency period of mesothelioma among a cohort of British asbestos workers (1978-2005). Br J Cancer. 2013;109(7):1965-73.

35. Marinaccio A, Montanaro F, Mastrantonio M, et al. Predictions of mortality from pleural mesothelioma in Italy: A model based on asbestos consumption figures supports results from age-period-cohort models. Int J Cancer. 2005;115(1):142-7.

36. Hodgson JT, Mc Elvenny DM, Darnton AJ, et al. The expected burden of mesothelioma mortality in Great Britain from 2002 to 2050. Br J Cancer. 2005;92(3):587-93. 